\title{
GAS CONTENT OF MARKARIAN STARBURST GALAXIES
}

\author{
RAFIK KANDALYAN \\ Byurakan Astrophysical Obs., 378433 Byurakan, Armenia \\ E-mail: rkandali@bao.sci.am,rafik@ipia.sci.am
}

\begin{abstract}
The main results of this study can be summarized as follows: (a) The $\mathrm{HI}$ and $\mathrm{CO}$ linewidths are well correlated. Interaction between galaxies has little influence on the $\mathrm{H} \mathrm{I}$ and $\mathrm{CO}$ line broadening. A rapidly rotating nuclear disk in the galaxy could lead to CO line broadening, while the $\mathrm{H}$ I line is less affected by the rotating disk. Molecular gas in Markarian galaxies is centrally concentrated. (b) For past and present star formation activity both $\mathrm{H} \mathrm{I}$ and $\mathrm{H}_{2}$ components of the gas are important. The atomic and molecular gas surface densities are well correlated with blue, FIR, and radio continuum surface brightnesses, but the $\mathrm{H}_{2}$ surface density is better correlated than that of the $\mathrm{H}$. The two gas phases are also connected. (c) In general, galaxies with UV-excess (Markarian galaxies) are not distinguished by star formation properties from non-UV galaxies, however some second order differences may exist, like the relation between atomic surface density and radio continuum surface brightness.
\end{abstract}

\section{Results}

Our original sample of Mkn-IRAS galaxies contains 155 objects (Kandalian et al. 1995). In order to investigate the gas properties of Mkn-IRAS galaxies in our sample we have extracted from the literature all objects which were detected in the ${ }^{12} \mathrm{CO}(1-0)$ line (till July, 1997). The total number of CO detected Mkn galaxies is 61 . Optical, FIR, H I and radio continuum data have been extracted from the literature (Martin et al. 1991,1997; Kandalian et al. 1997 and references therein). In order to study the gas kinematics of Mkn galaxies we have made a statistical investigation of the $\mathrm{H}$ I and $\mathrm{CO}$ linewidths, W. There is a good correlation between $W_{\mathrm{HI}}$ and $W_{\mathrm{CO}}$ (correlation coefficient $r=0.72$ at significance $p<0.0001$ ). Sofue et al. (1993) have suggested that tidal interaction could disturb the outermost but not 
innermost regions of a galaxy. As a conseauence $W_{\mathrm{H}}$ for paired+interacting galaxies will be much broader than that for isolated ones and no difference will be observed in $W_{\mathrm{CO}}$ between these two types of galaxies. Our analysis shows that there are no significant differences between $W_{\mathrm{H}_{\mathrm{I}}}$ and $W_{\mathrm{CO}}$ for isolated and paired+interacting galaxies, although $W_{\mathrm{H}}$ for the latter is slightly higher than that for the former. According to our data there are galaxies with $W_{\mathrm{HI}}-W_{\mathrm{CO}}<0$. It is likely that when $W_{\mathrm{CO}}>W_{\mathrm{HI}}$ it indicates the existence of a rapidly rotating nuclear disk in the galaxy, and as a consequence, rotation curves of these galaxies could have a peak in their central region $(<1 \mathrm{kpc})$. It could also be due to an outflow from the nuclear region (expanding molecular gas). The clumpy structure of molecular gas could also lead to CO line broadening.

We have compared gas-to-luminosity relations for 5 samples, namely optically selected nearby galaxies (Nearby); optically selected starburst galaxies (Starburst); Markarian galaxies detected by IRAS (UV-IRAS); IRAS selected galaxies (IRAS) and galaxies which belonged in clusters (Cluster). Several important conclusions have been drawn: (a) For all samples, FIR surface brightness is more tightly correlated with the $\mathrm{H}_{2}$ surface density than with $\mathrm{H}$ I surface density. (b) The tight correlation between $\mathrm{H}$ I surface density and FIR surface brightness for all samples indicates that the H I phase is also important for current or recent star formation. (c) Both phases of the gas are linked with indicators of past star formation, but the molecular phase does not now dominate in this relation for all samples as was the case for FIR emission. (d) There is tight correlation between $\sigma_{\mathrm{R}}$ and $\sigma_{\mathrm{H}_{2}}$ and for the "UV-IRAS" sample, the radio continuum surface brightness is also correlated with the H I surface density. (e) The galaxies with UV-excess are not distinguished in their star formation properties from other galaxies.

\section{References}

Kandalian R.A. et al. 1995, Afz, 38, 639

Kandalian R.A., Martin J.-M., Bottinelli L., \& Gouguenheim L. 1997, in preparation Martin J.-M. et al. 1991, $A \& A$ 245, 393

Martin J.-M., Kandalian R.A., Horellou C., Bottinelli L., \& Gouguenheim L. 1997, in preparation

Sofue Y. et al. 1993, PASJ, 45, 43 